\title{
Análisis de los Esquemas de Argumentación de Estudiantes de Secundaria en Ambientes de Geometría Dinámica
}

\author{
María del Carmen Fajardo Araujo \\ Víctor Larios Osorio
}

\author{
Facultad de Informática, Facultad de Ingeniería \\ Universidad Autónoma de Querétaro \\ e-mail: carmulita_@hotmail.com,vil@uaq.mx
}

\section{Resumen}

Este trabajo constituye un avance del proyecto de investigación doctoral enfocado a conocer los procesos cognitivos que intervienen cuando un alumno aprende a validar conjeturas con ayuda de una herramienta, en este caso el software de geometría dinámica, ya que este puede servir para hacer conjeturas sobre objetos geométricos, especificamente estos pueden jugar el rol de mediador en la transición entre la argumentación y la prueba, a través de la función de $\dot{\Xi}$ arrastre?, que abre nuevas rutas a los conocimientos teóricos dentro de un entorno concreto que sea significativo para los estudiantes. El analizar los argumentos que los alumnos dan, será un referente importante para llegar a la demostración, es decir suponemos que la argumentación antecede a la demostración.
\end{abstract}

\section{Introducción}

La necesidad de realizar un estudio sobre los procesos cognitivos que lleva a cabo un alumno para demostrar, viene apoyada por las exigencias sociales que son plasmadas en las Reformas Educativas que recientemente se han hecho para todos los niveles de escolaridad. De manera que los beneficios que tendría un individuo si se le instruye desde niveles básicos en la demostración, según Hanna [2] trascenderían a niveles superiores en carreras donde hay alto contenido matemático. Otra razón para centrar el interés en la demostración en educación básica, se apoya en las recomendaciones a favor de ésta, del National Council of Teachers of Mathematics (NCTM), que enfatizan incluir al razonamiento y la demostración como parte consistente de la experiencia matemática durante toda la escolaridad [5].

Para educación secundaria se espera que los alumnos dentro del campo formativo de pensamiento matemático, usen el razonamiento como herramienta fundamental para la solución de problemas; que vayan del razonamiento intuitivo al deductivo, formulen y validen conjeturas, busquen argumentos para validar procedimientos y resultados, comuniquen, analicen e interpreten procedimientos de resolución [6]; en la competencia de validar procedimientos y resultados los alumnos adquirirán confianza suficiente para explicar y justificar los procedimientos y las soluciones encontradas, mediante argumentos a su alcance que se orienten al razonamiento deductivo y la demostración formal, enfatizando explícitamente el razonamiento como una vía para alcanzar esos objetivos. Entendemos entonces que se trata de una adquisición gradual de habilidades y conocimientos que permitan al alumno transitar 
en procesos distintos como los demostrativos, argumentativos, etc. A pesar de que algunos hacen la separación.

\section{Referente Teórico}

Tomando en cuenta las clasificaciones de Balacheff, los alumnos de nivel secundaria sólo dan explicaciones de lo que hacen, en la mayoría de los casos esas explicaciones cumplen parcialmente con algunas características que tienen los argumentos matemáticos (los que hace la comunidad matemática), por lo que resulta necesario caracterizar el tipo de argumentos que emergen en el salón de clases. González y Larios [4] plantearon una serie de elementos que pudieran ayudar a la caracterización; todo argumento escolar entonces debería cumplir con lo siguiente: x1. Hacer referencia a un hecho matemático. 2. Tener como función primaria convencer a sí mismo y a otros para proporcionar una explicación al hecho matemático. 3. Las formas de comunicación utilizadas deben ser conocidas por los miembros de la comunidad escolar, o aprendidas por ellos. 4. Los enunciados utilizados deberán ser aceptados por la comunidad escolar, ya sea explícita o implícitamente. 5. La serie de argumentos deberá organizarse con base en formas de razonamiento válidas o correctas.

El modelo estructural de Toulmin [7] que propone una serie de términos (datos, garantías y conclusiones) necesarios para comprobar la validez de todo argumento, será un referente para determinar un modelo adecuado al contexto escolar para conocer cómo y de qué clase son los argumentos proporcionados por los alumnos en las actividades propuestas, la figura siguiente relaciona los términos base mencionados:

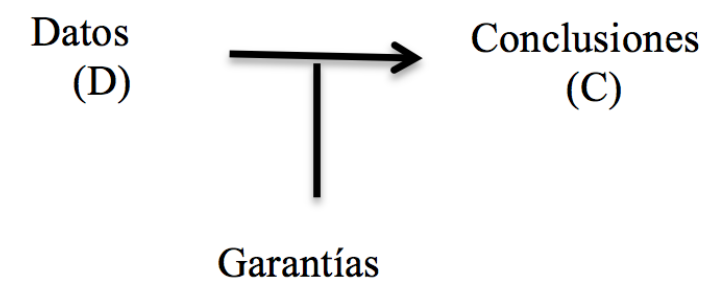

(G)

Figura 1. Modelo de Toulmin

El anterior modelo sería la etapa final a la que un alumno llegaría conforme avanza en el aprendizaje de las matemáticas, es decir este modelo refiere a todo argumento que se desarrolla en la matemática misma, pero que no funciona totalmente con los alumnos que no se formarán como matemáticos profesionales, entonces habrá que agregar otros elementos que ayuden a los alumnos a generar argumentos en ámbitos escolares, éstos podrían ser los modelizadores (M) y las condiciones excepcionales (E) que pueden servir para aceptar o rechazar las conclusiones dependiendo de la situación, la figura muestra cómo esos elementos pudieran apoyar los argumentos de los alumnos.

La identificación de los términos que componen los argumentos de los alumnos de secundaria, nos permitirá reconocer en qué tipo de esquema argumentativo están. Los esquemas 


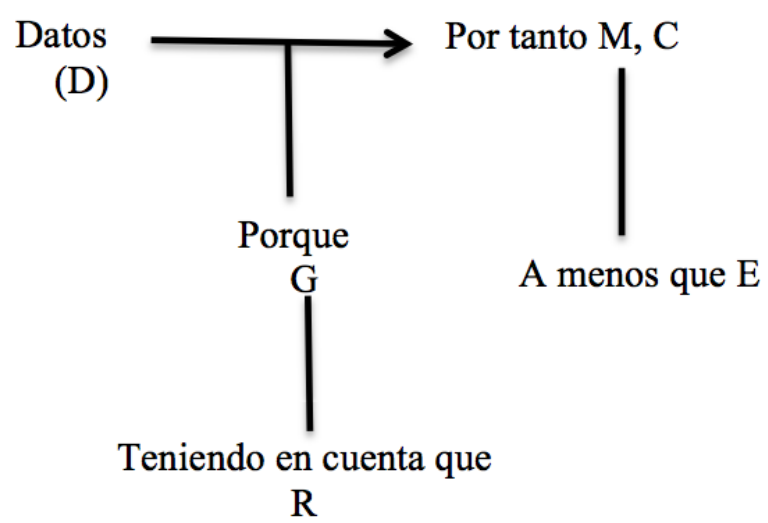

Figura 2. Modelo Escolar Argumentativo

de argumentación son tomados de Flores [1] quien de acuerdo al tipo de razonamiento usado por el individuo en una práctica argumentativa los denomina como:

Autoritarios, donde sus argumentaciones se apoyan de las afirmaciones hechas por una autoridad (profesor, libro de texto).

Simbólicos, donde se utiliza un lenguaje matemático y símbolos de manera superflua y poco consistente sin llegar realmente a las conclusiones deseadas, el sujeto puede mencionar conceptos poco claros o inventados.

Fácticos, se hace un recuento de lo que se hizo o se repiten hechos evidentes de una situación a manera de explicación o justificación de algún resultado, como una serie de pasos que parecen un algoritmo.

Empíricos, apoyo de hechos físicos o en un dibujo, donde este constituye un argumento por sí mismo y no u apoyo para visualizar el argumento.

Analíticos, se sigue una cadena deductiva, sin que por ello se llegue forzosamente a una conclusión válida.

\section{La propuesta de actividades}

Las actividades que se diseñen para el experimento de enseñanza tendrán la intencionalidad de desarrollar en los alumnos habilidades como la visualización, la formulación de conjeturas, el razonamiento deductivo, etc., elementos que anteceden al proceso de argumentación. Se pretende que las actividades sigan la unidad cognitiva de los teoremas [3]:

Puesto que apenas se están diseñando las actividades que se usarán en el experimento de enseñanza, se describe de manera general la estructura que tendrán y parcialmente se ejemplifica una de ellas, de acuerdo a las etapas mencionadas anteriormente; los alumnos con ayuda del software GeoGebra han de realizar las construcciones y observar regularidades con el fin de elaborar justificaciones a sus respuestas. En términos generales las actividades se desarrollarán en seis etapas: 


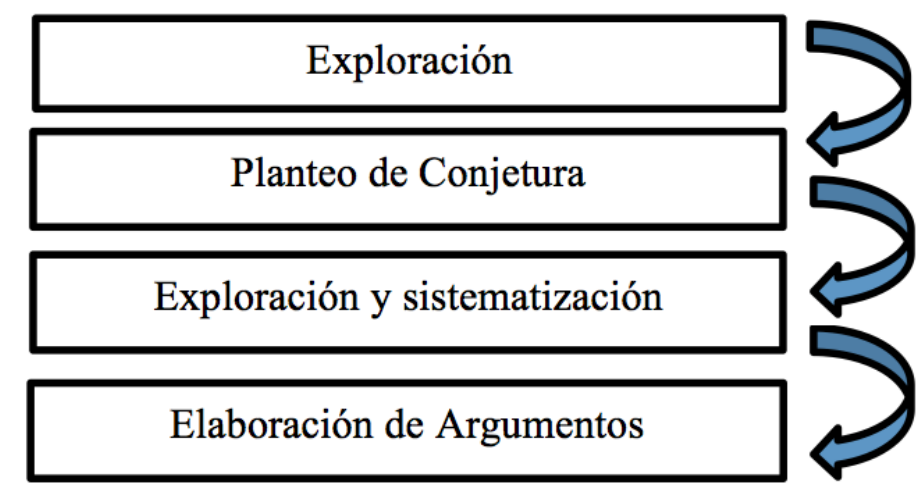

Figura 3. Unidad Cognitiva de los Teoremas

1. Planteamiento de la situación, que se hará a través de una consigna, usando principalmente textos para que los alumnos realicen la construcción en el software o bien se les proporcionará un archivo ya creado.

2. Construcción de la situación, se les pedirá a los alumnos en la mayoría de las actividades, que realicen una construcción con el software, intentando apoyarles en la corrección de las mismas, dada la inexperiencia en el manejo del software.

3. Exploración de la situación, se dejará a los alumnos parcialmente libres, será una libertad dirigida, puesto que observaran hechos.

4. Explicitación de propiedades observadas, al terminar cada construcción se solicitará a los alumnos que expliciten las propiedades observadas, incluso pudieran también escribir las acciones realizadas como parte de la exploración.

5. Justificación de propiedades, se pedirá a los alumnos que justifiquen las propiedades y que gradualmente dichas justificaciones tengan una estructura deductiva.

6. Conclusión, se les proporcionará a los alumnos una conclusión de las propiedades estudiadas, permitiendo llevarlas a la institucionalización, un consenso entre alumnos y profesor.

La actividad siguiente ejemplifica cómo serán construidas las demás, tiene la intencionalidad de que el alumno justifique las características de rectas que tocan en dos puntos a la circunferencia, que la tocan en un punto o que no la tocan.

\section{Reflexiones}

El proceso de argumentación en los ámbitos escolares es gradual y necesita implementarse desde niveles básicos de escolaridad (primaria, secundaria), no debe omitirse puesto que el desarrollo del razonamiento no sólo impacta en la escuela, sino que tiene que ver en otros 
Exploración: construcción de una recta tangente a una circunferencia desde un punto de ésta y observación de propiedades.

\begin{tabular}{l} 
Planteo de Conjeturas: afirmación sobre la recta $t$ es tangente a la \\
circunferencia C, en P. \\
$\begin{array}{l}\text { Exploración y Sistematización: construcción a partir de posiciones relativas } \\
\text { entre rectas y una circunferencia y entre circunferencias. }\end{array}$ \\
$\begin{array}{l}\text { Elaboración de Argumentos: propuesta de un procedimiento de construcción y } \\
\text { argumentos que lo apoyen. }\end{array}$ \\
\hline
\end{tabular}

contextos, entonces es necesario diseñar actividades que permitan a los alumnos adquirir habilidades que vayan mejorando sus argumentaciones, que progresivamente abandonen el uso de términos inventados para referirse a los objetos matemáticos, que sean capaces de elaborar justificaciones no porque el libro las dice, o porque un profesor las menciona (creencia de que lo dicho por el profesor es irrefutable y funciona como argumento para un resultado). El profesor tomando en cuenta el nivel cognitivo de los alumnos, los conducirá a alcanzar un tipo de argumentación que funciona en los contextos escolares, pero que constituye la antesala de las argumentaciones que han de darse en carreras profesionales con fuerte contenido matemático.

\section{Referencias}

[1] Flores, Á. (2007). Esquemas de Argumentación en Profesores de Matemáticas de Bachillerato. Educación Matemática, $63-98$.

[2] Hanna, G. (10-15 de mayo de 2009). International Commission on Mathematical instruction. Recuperado el 28 de abril de 2014, de ICMI 19: www.mathunion.org

[3] Larios, V. (2006). Demostrar es un problema o el problema es demostrar. Querétaro: UAQ. 
[4] Larios, V., \& González, N. (2012). Justificaciones en la Geometría Dinámica de Secundaria. Alemania: Academía Española.

[5] Mathematics, N. C. (2000). Principles and standards for school mathematics. EEUU: NCTM.

[6] SEP. (2011). Planes de Estudios 2011. México D.F.: Sep.

[7] Toulmin, S. (2003). The uses of argument. Barcelona: Ediciones Península . 